\title{
Postural Stability in Obese Preoperative Bariatric Patients Using Static and Dynamic Evaluation
}

\author{
Gabriel M. Pagnotti ${ }^{a} \quad$ Amna Haider ${ }^{a} \quad$ Ariel Yang ${ }^{a}$ Kathryn E. Cottell ${ }^{b}$ \\ Catherine M. Tuppo ${ }^{b}$ Kai-Yu Tong ${ }^{c}$ Aurora D. Pryor ${ }^{b}$ Clinton T. Rubin ${ }^{a}$ \\ M. Ete Chan ${ }^{a}$ \\ aDepartment of Biomedical Engineering, Stony Brook University, Stony Brook, NY, USA; \\ ${ }^{\mathrm{b}}$ Bariatric and Metabolic Weight Loss Center, Stony Brook Medicine, Stony Brook, NY, USA; \\ 'Department of Biomedical Engineering, The Chinese University of Hong Kong, Shatin, \\ Hong Kong
}

\section{Keywords}

Bariatric intervention · Obesity · Musculoskeletal health · Postural instability · Fall risk

\begin{abstract}
Introduction: Globally, 300 million adults have clinical obesity. Heightened adiposity and inadequate musculature secondary to obesity alter bipedal stance and gait, diminish musculoskeletal tissue quality, and compromise neuromuscular feedback; these physiological changes alter stability and increase injury risk from falls. Studies in the field focus on obese patients across a broad range of body mass indices $\left(\mathrm{BMI}>30 \mathrm{~kg} / \mathrm{m}^{2}\right.$ ) but without isolating the most morbidly obese subset $\left(\mathrm{BMI} \geq 40 \mathrm{~kg} / \mathrm{m}^{2}\right)$. We investigated the impact of obesity in perturbing postural stability in morbidly obese subjects elected for bariatric intervention, harboring a higher-spectrum BMI. Subjects and Methods: Traditional force plate measurements and stabilograms are gold standards employed when measuring center of pressure (COP) and postural sway. To quantify the extent of postural instability in subjects with obesity before bariatric surgery, we assessed 17 obese subjects with an average BMI of $40 \mathrm{~kg} / \mathrm{m}^{2}$ in contrast to 13 nonobese subjects with an average BMI of $30 \mathrm{~kg} / \mathrm{m}^{2}$. COP and postural sway were measured from static and dynamic tasks. Involuntary movements were measured when patients performed static stances, with eyes either opened or closed. Two additional voluntary movements were measured when subjects performed dynamic, upper torso tasks with eyes opened. Results: Mean body weight was $85 \%(p<0.001)$ greater in obese than nonobese subjects. Following static balance assessments, we observed greater sway displacement in the anteroposterior (AP) direction in obese subjects with eyes open $(87 \%, p<0.002)$ and eyes closed $(76 \%, p=0.04)$ versus nonobese subjects. Obese subjects also exhibited a higher COP veloc-
\end{abstract}

\section{Karger"}


Pagnotti et al.: Postural Instability in Bariatric Patients

ity in static tests when subjects' eyes were open $(47 \%, p=0.04)$. Dynamic tests demonstrated no differences between groups in sway displacement in either direction; however, COP velocity in the mediolateral $(\mathrm{ML})$ direction was reduced $(31 \%, p<0.02)$ in obese subjects while voluntarily swaying in the AP direction, but increased in the same cohort when swaying in the ML direction (40\%, $p<0.04)$. Discussion and Conclusion: Importantly, these data highlight obesity's contribution towards increased postural instability. Obese subjects exhibited greater COP displacement at higher AP velocities versus nonobese subjects, suggesting that clinically obese individuals show greater instability than nonobese subjects. Identifying factors contributory to instability could encourage patient-specific physical therapies and presurgical measures to mitigate instability and monitor postsurgical balance improvements.

(C) 2020 The Author(s)

Published by S. Karger AG, Basel

\section{Introduction}

Involuntary falls in sedentary [1] and elderly [2] populations contribute significantly towards morbidity and early mortality [3], specifically when compounded by obesity [4-6]. As a consequence of aging, diminished musculoskeletal tissue quality [7, 8] and degraded neuronal feedback mechanisms tarnish muscle tone and motor control responses [9] that are necessary for maintaining balance $[10,11]$. Sarcopenia coupled with obesity further impairs balance [12], directing inertial forces away from the body's center of mass (COM). Thus, obese individuals might involuntarily stray from a perceived point of balance [4], especially while in motion. Increased momentum upon impact and greater body mass further potentiates a greater degree of injury. Comorbidities associated with aging in combination with obesity contribute heavily towards instability [13] and subsequent fall risk statistics. Quantifying differences in stability could lead to more effective strategies to mitigate the incidence of falls in the obese population [14].

Global rates of obesity have risen dramatically in recent years, with 13\% of adults (aged $>18$ years) and 41 million children [15] contributing to this epidemic in 2014 [16]. Within the U.S. alone, $36 \%$ of adults have a body mass index (BMI) $\geq 30 \mathrm{~kg} / \mathrm{m}^{2}$, accompanied by USD 147 billion medical expenditures [17]. Individuals with obesity categorically have a BMI $\geq 40 \mathrm{~kg} /$ $\mathrm{m}^{2}$ (class III) or $\geq 35 \mathrm{~kg} / \mathrm{m}^{2}$ (class II) accompanied by a comorbidity [18], only then becoming eligible candidates for most bariatric surgical interventions [19]. Elderly and juvenile obesity compromises gait kinetics [20], which is exacerbated by debilitating joint stress [21] that derives from reduced mobility and cartilage damage [22]. Treatment strategies ranging from diet control and balance training [23] to surgical procedures [24] are employed to counter these effects and normalize center of pressure (COP) velocity [25] and mobility [26].

In order to measures the degree of fall risk, both static and dynamic balance assessments are important. Posturography [27], utilizing force plates, upright motion tests, and stabilograms aids in visualizing spontaneous sway [28] and gauge fall predictor risk in the elderly $[29,30]$ and those with neurological impairment [31]. Historically, the Romberg test visually aids in evaluating postural stability (specifically, diagnosing ataxia and upright disequilibrium [32]) through series of exercises involving static [33] and dynamic stability measurements. Studies have assessed stability in a broad range of obese patients through gait analyses and static and dynamic posturography. While static evaluations are strictly limited to a standing evaluation with eyes open and eyes closed, dynamic testing can be performed through various modalities. For example, cases of pediatric obesity have exhibited increased instability when subjects were challenged to perform a lunge test [34]; some of these stability limitations derive from altered joint mechanics and poor musculature and flexibility about the foot [35]. Our study employed balance assessments to measure involuntary sway and active move-

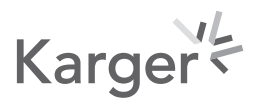


Pagnotti et al.: Postural Instability in Bariatric Patients

ments, thereby assessing to what degree measures of stability in obese subjects deviated from the leaner, nonobese body type. However, to date, minimal rigorous assessments quantifying both static and dynamic COP measurements in patients qualified for bariatric surgery categorized exclusively in the higher spectrum of obesity class have been performed $[25,36]$.

We hypothesized that candidates elected for bariatric surgery, who are categorized as obese along the higher end of the BMI spectrum, exhibit characteristically greater COP sway magnitudes and velocities away from the neutral center point when compared to nonobese subjects. Brief static and dynamic motion tasks were employed to contrast the postural stability of 17 subjects categorized with obesity to 13 nonobese subjects. As individuals afflicted by obesity have inadequate upright balance musculature (involuntary sway about the midline) $[37,38]$ and normal locomotion [39], challenging obese subjects with static [40] and dynamic tasks was critical to identifying which element(s) of their balance repertoire had become compromised.

\section{Subjects and Methods}

\section{Subject Recruitment}

This research comprised a joint collaboration between the Division of Bariatric, Foregut and Advanced Gastrointestinal Surgery housed within the Department of Surgery at Stony Brook Medicine and the Biomedical Engineering Department at Stony Brook University. Recruitment of obese subjects was performed at Stony Brook's Bariatric and Metabolic Weight Loss Center while nonobese subjects were recruited from Stony Brook University. As determined based on findings derived from force plate measurements in other published studies that compared stability in obese patients to that in normal-weight subjects, 30 subjects were recruited over 3 months and grouped as either obese $(n=17)$ or nonobese $(n=13)$ [41, 42]. Patient criteria included the following:

Exclusion Criteria. Subjects who (a) were nonambulatory or required walking assistance, (b) did not understand the potential benefits and study risks, (c) did not provide consent, (d) required use of a prosthetic, walker, wheelchair, or cane, and/or (e) were pregnant.

Inclusion Criteria. Obese subjects: (a) male or female, (b) aged 18-65 years, (c) Englishspeaking, (d) without lower limb prostheses, (e) with no evidence of impaired gait due to illness, fracture, or existing wound, (f) classified as obese (BMI $\geq 30 \mathrm{~kg} / \mathrm{m}^{2}$ ), and /or (g) participating in the Division of Bariatric, Foregut and Advanced Gastrointestinal Surgery weight loss program at Stony Brook University. Nonobese subjects: (a) male or female, (b) aged 18-65 years, (c) English-speaking, (d) without lower limb prostheses, (e) with no evidence of impaired gait due to illness, fracture, or existing wound, and/or (f) classified as nonobese $\left(\mathrm{BMI}<30 \mathrm{~kg} / \mathrm{m}^{2}\right)$.

\section{Stability Measurements}

Subjects were monitored and permitted to dismount the platform if discomfort was experienced, in which case data would be recaptured. Tasks were performed with subjects standing in a fixed position atop a piezoelectric force plate (9286AA; Kistler, Winterthur, Switzerland) fitted with triaxial strain-gauge transducers beneath each footing, which measured the body's net applied downward force. Measurements were recorded using an eight-channel amplifier, an analog-digital converter, and the Bioware software (v.3.2.6.104; Kistler, USA). Tasks were recorded over $15-$ s intervals at a $30-\mathrm{Hz}$ sampling frequency. Subject postural stability was quantified by shifts (measured in $\mathrm{mm}$ ) in radial COP as well as root mean square velocity $\left(\mathrm{V}_{\mathrm{RMS}}\right.$ ) along the anteroposterior (AP) and mediolateral (ML) directions. Subjects were instructed to stand upright but relaxed with feet facing forward, heels

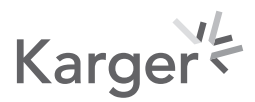


Pagnotti et al.: Postural Instability in Bariatric Patients

aligned three inches from the rear edge of the platform while maintaining a shoulder-width stance (shown in online suppl. Fig. 1; see www.karger.com/doi/10.1159/000509163 for all online suppl. material). Here, we utilized posturography as a quantitative tool to evaluate subjects' vestibulospinal reflex to maintain balance in either static or dynamic tasks. Below we summarize the definitions of key parameters measured in stability studies [27, 43, 44]: COP: the vertical ground reaction force vector measured with the body positioned over the feet as they rest on a surface. Mean sway displacement: the average traveling distance (excursion) of the COP in either the AP or ML direction. $\mathrm{V}_{\mathrm{RMS}}$ : the velocity of the subject's COP movement.

Measurements were gathered by employing four kinematic analyses, which consisted of two consecutive static followed by two consecutive dynamic motion posturography techniques. The subject's weight and age were recorded immediately prior to initiating the first task. Postural tasks (note that force plates were normalized to each subject's controlled, static stance at the beginning of each data acquisition): (I) static: eyes open, standing still; (II) static: eyes closed, standing still; (III) dynamic: eyes open, intentionally swaying fore and aft (AP); (IV) dynamic: eyes open, intentionally swaying from right to left (ML).

Subjects were instructed to perform tasks without dismounting the platform or lifting their heels unless they felt a fall was imminent. Subjects were prompted with verbal cues at the beginning and completion of assigned tasks. Compiled force plate measurement data were then processed through a custom MATLAB (MathWorks Inc., Natick, MA, USA) program modified from Muir et al. [45], with output peak AP and ML displacements and velocities derived from involuntary sway acquired from each prescribed task. To ensure subject safety while maintaining balance during a voluntary dynamic task (tasks III and IV), each participant was permitted freedom to sway in the assigned direction according to their personal degree of comfort without restriction to a preassigned speed or frequency. Employing "stabilograms," subjects' range of motion about a normalized reference mark on the force plate $z$ axis was recorded as the COP, which was then continuously tracked across the $x$ and $y$ axes (online suppl. Fig. 2). This measure would change depending on the duration, magnitude, and velocity of the displacement of weight further from the base of the heel.

\section{Statistical Analysis}

The statistical analysis was generated using Prism (v.8; GraphPad Software Inc., San Diego, CA, USA) statistical software. In total, 30 subjects were recruited, and of these 17 obese and 13 nonobese patients were evaluated. A two-tailed Student $t$-test was performed to compare stability and subject data between both groups. $p$ values $\leq 0.05$ were considered statistically significant. All data are presented as mean \pm standard deviation.

\section{Results}

Subject Age and Weight Comparisons (Fig. 1)

The ages of nonobese ( $43 \pm 13$ years) subjects did not significantly deviate from those of obese subjects ( $48 \pm 12$ years). The mean BMI of obese subjects $\left(45.4 \pm 9.25 \mathrm{~kg} / \mathrm{m}^{2}\right)$ was $85 \%$ $(p<0.001)$ greater than that of nonobese subjects $\left(24.85 \pm 2.847 \mathrm{~kg} / \mathrm{m}^{2}\right)$.

\section{Stability Assessments}

For each task, between-groups comparisons were made to compare mean sway displacement along the AP and ML axes (independently), COP sway, and $\mathrm{V}_{\mathrm{RMS}}$ along the AP and ML axes.

\section{Karger's}



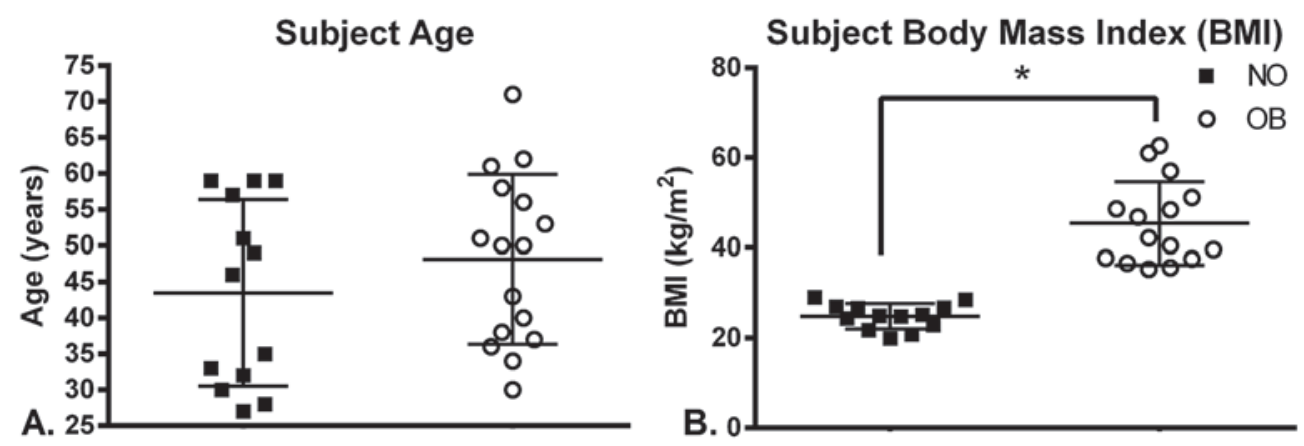

Fig. 1. Subjects' ages spanned 30-70 years and were matched between groups. Nonobese (NO) subjects presented with a mean $\mathrm{BMI}<30 \mathrm{~kg} / \mathrm{m}^{2}$, while subjects identified as obese (OB) had a measured BMI $\geq 30 \mathrm{~kg} / \mathrm{m}^{2}$. Data are presented as mean \pm standard deviation (NO: $n=13$; OB: $n=17$ ). ${ }^{*} p<0.05$ (two-tailed Student $t$ test).

Task I (Static Stance: Eyes Open) (Fig. 2). Mean sway displacements of obese subjects in the AP and ML directions were $87 \%(p<0.002)$ and $97 \%(p<0.01)$ greater, respectively, than those of nonobese ones. Isolating forward and backward COP displacement in the AP direction showed 67\% $(p<0.005)$ and 98\% $(p<0.005)$ greater displacement, respectively, in obese subjects than in nonobese ones. ML COP displacement to the subjects' right and left was $87 \%$ $(p<0.009)$ and $88 \%(p<0.003)$ greater, respectively, in obese subjects than in nonobese ones. AP root mean square (RMS) and ML RMS COP displacements in obese subjects were $79 \%(p<0.003)$ and $100 \%(p<0.007)$ greater, respectively, than RMS COP displacements measured from nonobese ones. COP $\mathrm{V}_{\mathrm{RMS}}$ for involuntary AP sway was $47 \%(p<0.05)$ greater in magnitude in obese subjects than in nonobese ones, but no differences in COP $V_{\text {RMS }}$ were observed in the ML direction. The maximum AP COP $V_{\text {RMS }}$ was $72 \%(p<0.03)$ greater in obese subjects, but no differences were observed in the other COP $\mathrm{V}_{\mathrm{RMS}}$ comparisons.

Task II (Static Stance: Eyes Closed) (Fig. 3). Throughout the duration of task II subjects were instructed to keep their eyes closed. Subject sway magnitude for both the obese and nonobese group was approximately twice the value in task II than for their respective counterparts in task I. Mean AP sway displacement of obese subjects was $76 \%$ greater $(p<0.005)$ than in nonobese ones, but no differences were observed in the ML direction. Obese subjects' forward COP displacements along the AP axis were 63\% $(p<0.03)$ greater than readings taken from nonobese ones, which contributed to a $61 \%(p<0.007)$ greater RMS COP in the AP direction. No differences were observed in backward COP displacement in the AP direction, nor were differences detected across the left or right ML COP displacement. The $\mathrm{V}_{\mathrm{RMS}}$ in the AP direction of obese individuals trended $41 \%$ ( $p=$ nonsignificant) greater in magnitude than in the nonobese counterparts, translating to a $49 \%(p<0.05)$ increase in the maximum COP $\mathrm{V}_{\mathrm{RMS}}$ in the AP direction of obese subjects relative to nonobese ones. No differences in $\mathrm{V}_{\mathrm{RMS}}$ magnitude in the ML direction were detected between groups.

Task III (Dynamic Stance AP Sway: Eyes Open) (Fig. 4). Obese subjects were able to sway in the AP direction to the same extent as nonobese ones when instructed. Mean AP and ML sway displacements were not different between groups. Neither forward nor backward COP displacements in the AP direction nor left and right COP displacements differed in readings taken from either subject cohort. $\mathrm{V}_{\mathrm{RMS}}$ in the AP direction did not differ in obese individuals as compared to nonobese ones; however, in contrast to the previous tasks, ML $V_{\text {RMS }}$ was $31 \%$ 


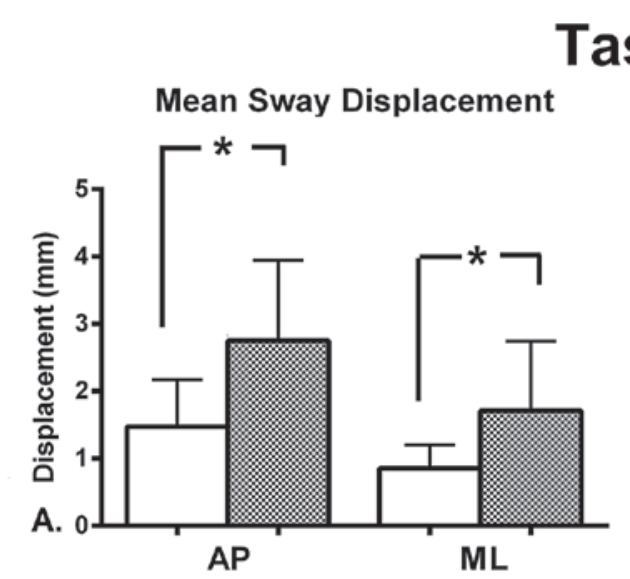

B. 0
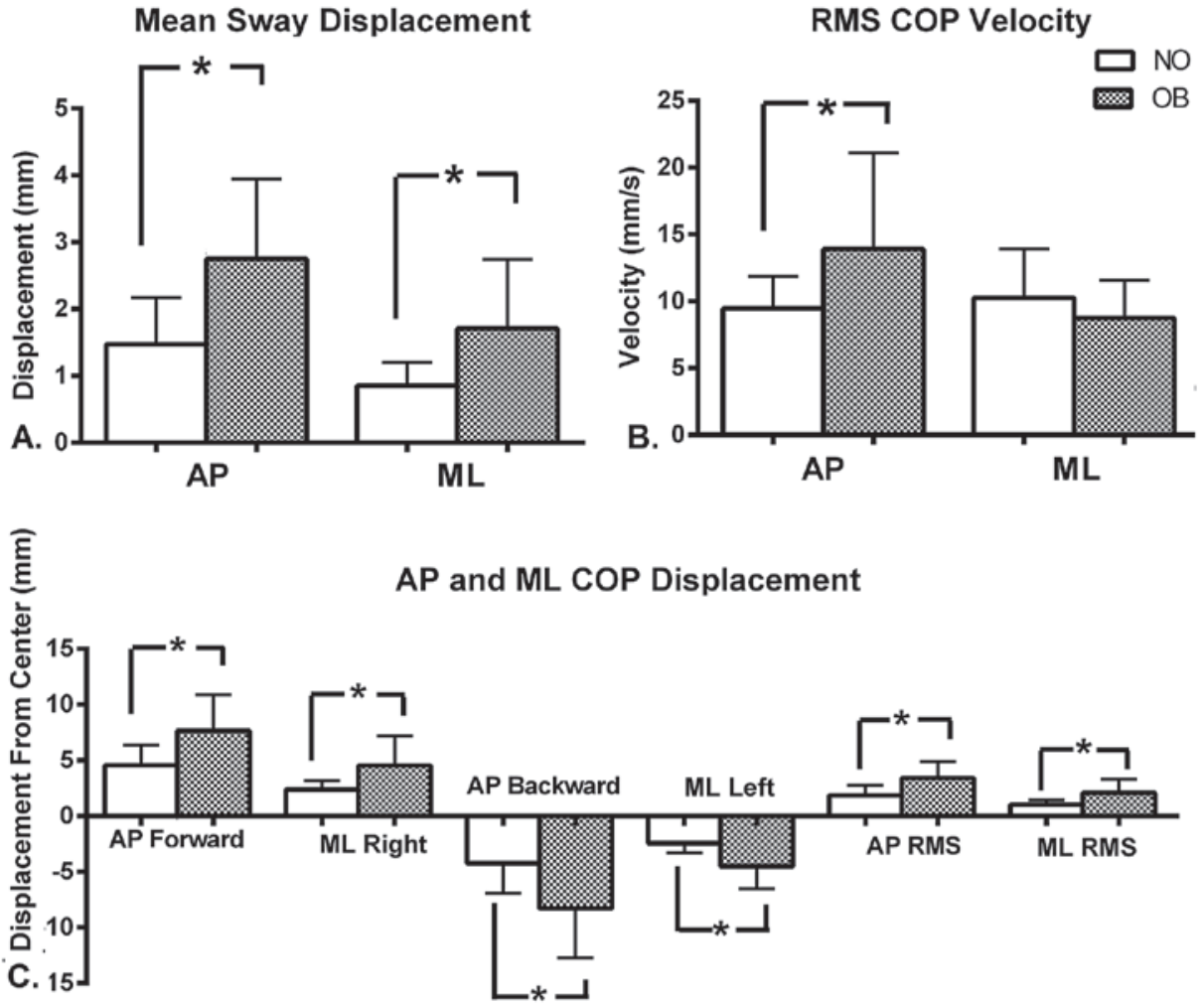

AP and ML Max and Min COP Velocities

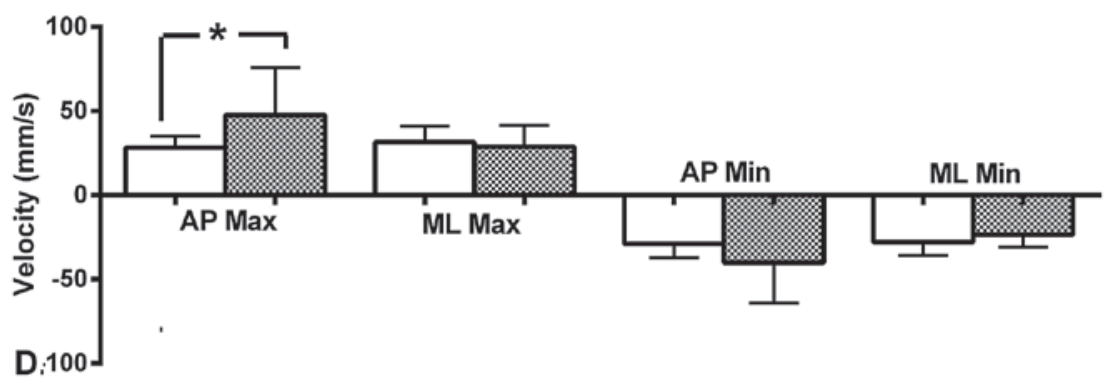

Fig. 2. Task I force plate measurements. Subjects were instructed to remain in a static stance with eyes open. A Both mean AP and ML sway displacements were significantly greater in obese (OB) than in nonobese (NO) subjects. B RMS COP velocities were significantly greater in OB subjects along the AP axis. C Displacements from the COP in OB subjects exceeded those of NO subjects in all directions. D COP velocity along the AP axis was significantly greater in OB subjects. Data are presented as mean \pm standard deviation (NO: $n=13$; OB: $n=17$ ). ${ }^{*} p<0.05$ (two-tailed Student $t$-test). AP, anteroposterior; COP, center of pressure; ML, mediolateral; RMS, root mean square. 


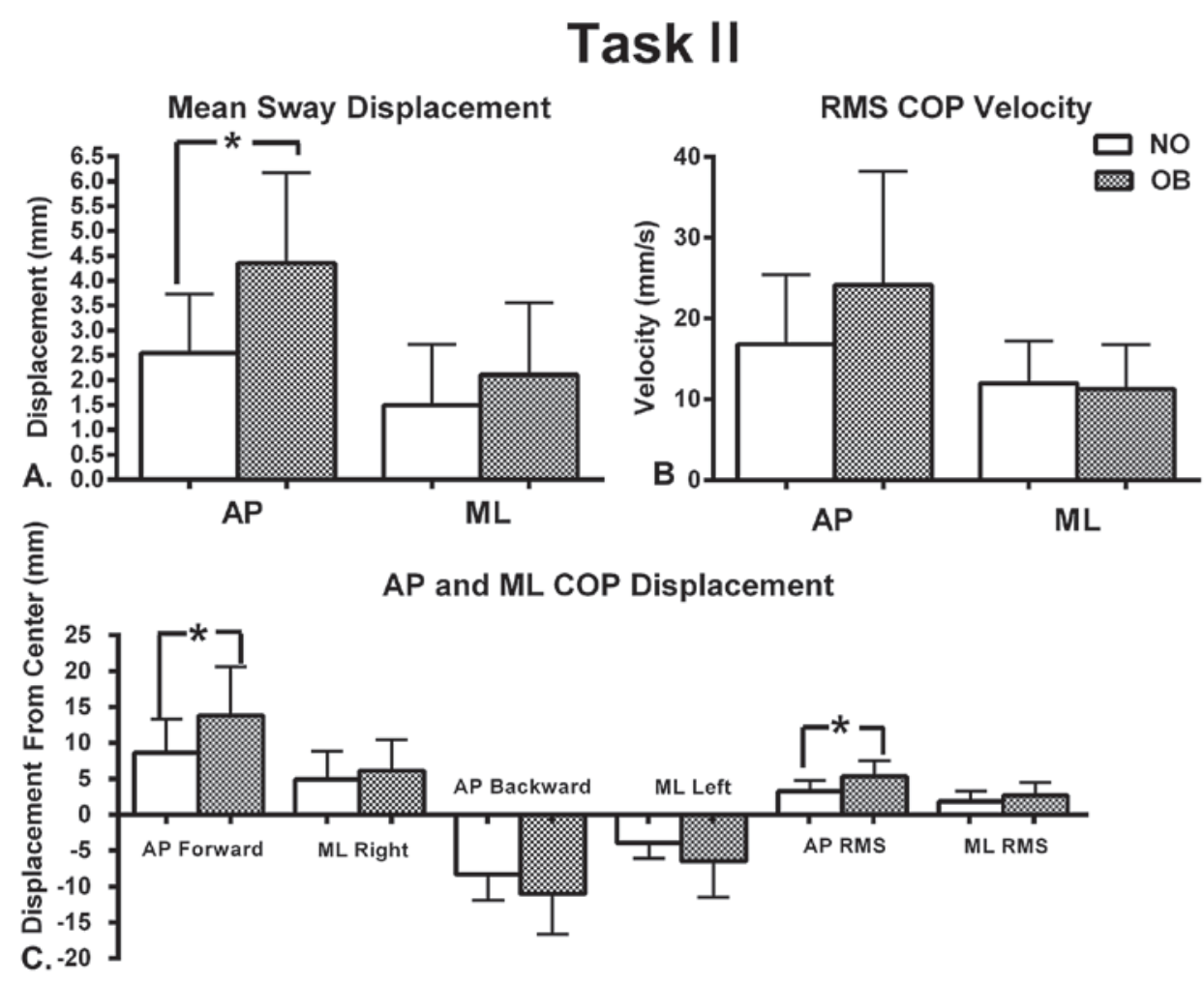

AP \& ML Max and Min COP Velocities

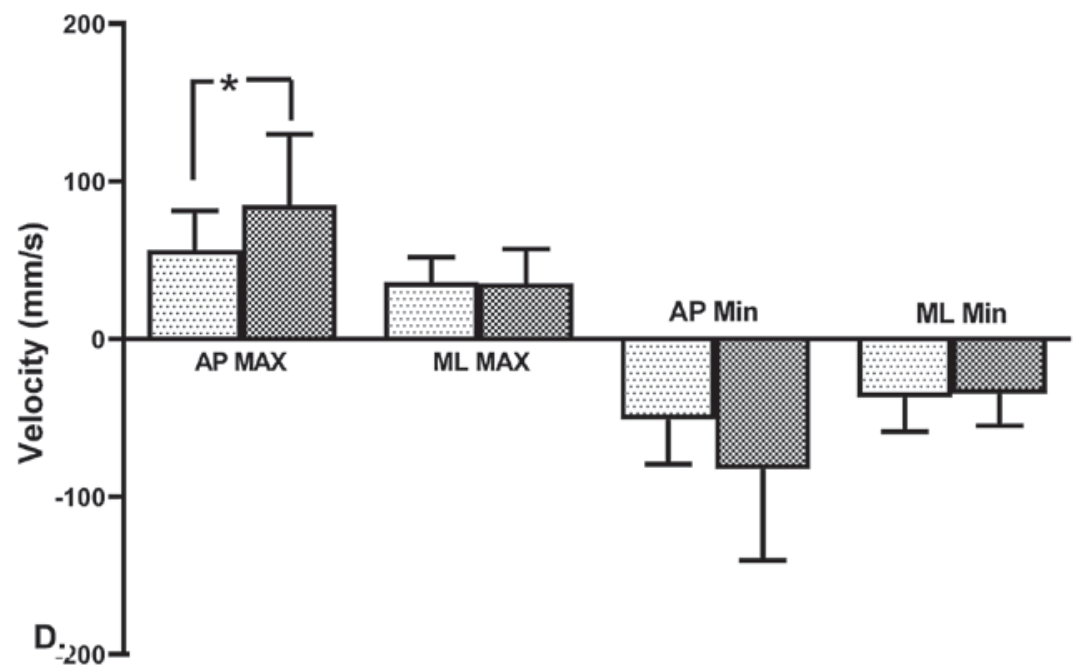

Fig. 3. Task II force plate measurements. Subjects were instructed to remain in a static position with eyes closed. A AP sway displacement was significantly greater in obese (OB) than in nonobese (NO) subjects; however, the displacement velocity from the COP was not significantly greater in OB compared to NO subjects (B). This contrasts the differences observed in the eyes open task (task I), perhaps due to a visual overestimation of imbalance, as suggested by the significant forward AP displacement (C) and velocity (D). Data are presented as mean \pm standard deviation (NO: $n=13$; OB: $n=17$ ). ${ }^{*} p<0.05$ (two-tailed Student $t$-test). AP, anteroposterior; COP, center of pressure; ML, mediolateral; RMS, root mean square. 


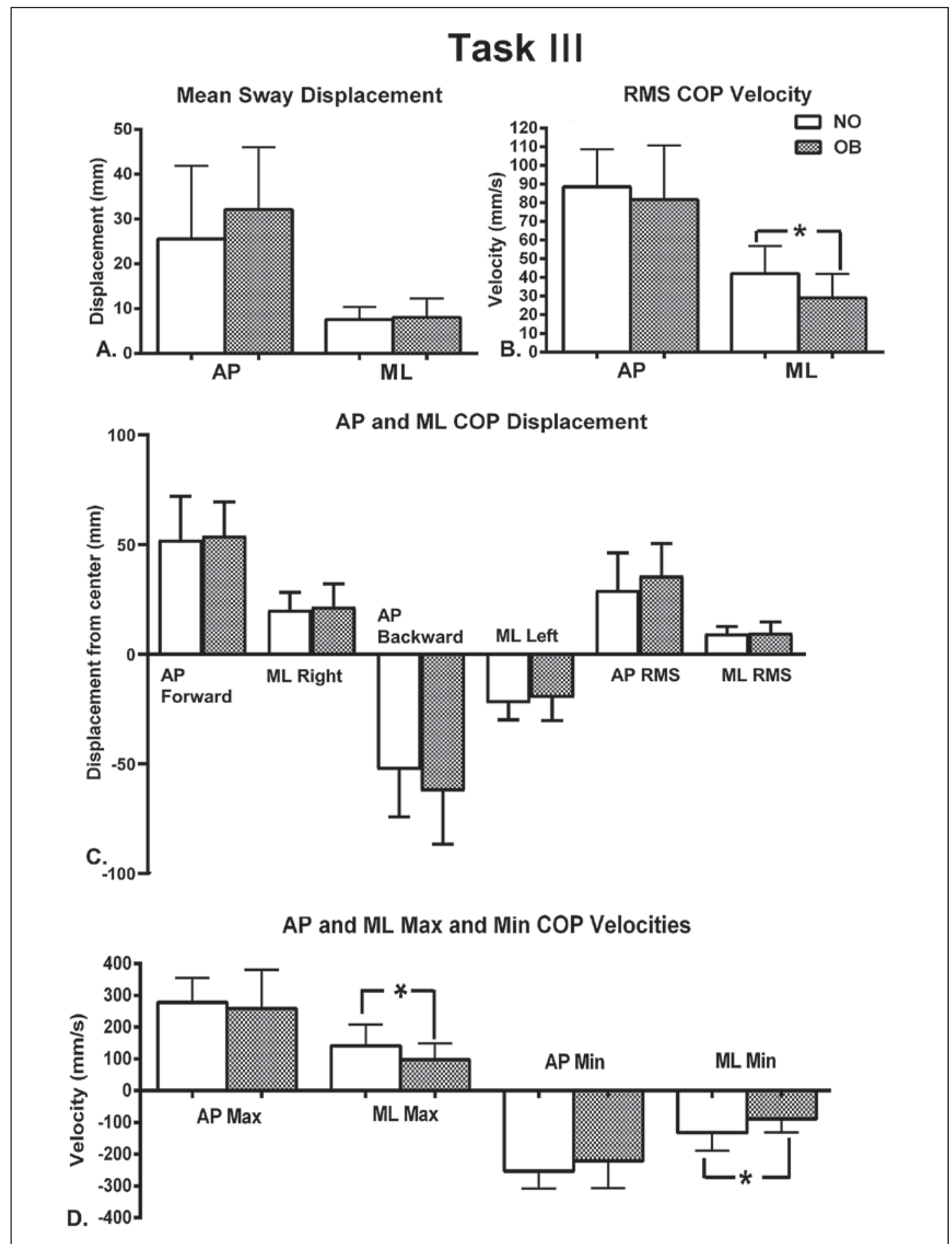

Fig. 4. Task III force plate measurements. Subjects were instructed to repeatedly sway both fore and aft along the AP axis (for $15 \mathrm{~s}$ ) of the force plate while keeping eyes open. Displacements (A) and velocities (B) throughout this task remained near identical between groups. Despite the minimal displacement having occurred within the confines of the ML direction (C), the velocity of the obese (OB) group was significantly lower along the ML axis (D). What these findings may allude to is an increased level of control when the torso is in motion versus when stationary even though $\mathrm{OB}$ subjects appear slower in traversing the fore-aft motion than nonobese (NO) subjects. Data are presented as mean \pm standard deviation (NO: $n=13$; OB: $n=17$ ). ${ }^{*} p<0.05$ (twotailed Student $t$-test). AP, anteroposterior; COP, center of pressure; ML, mediolateral; RMS, root mean square. 


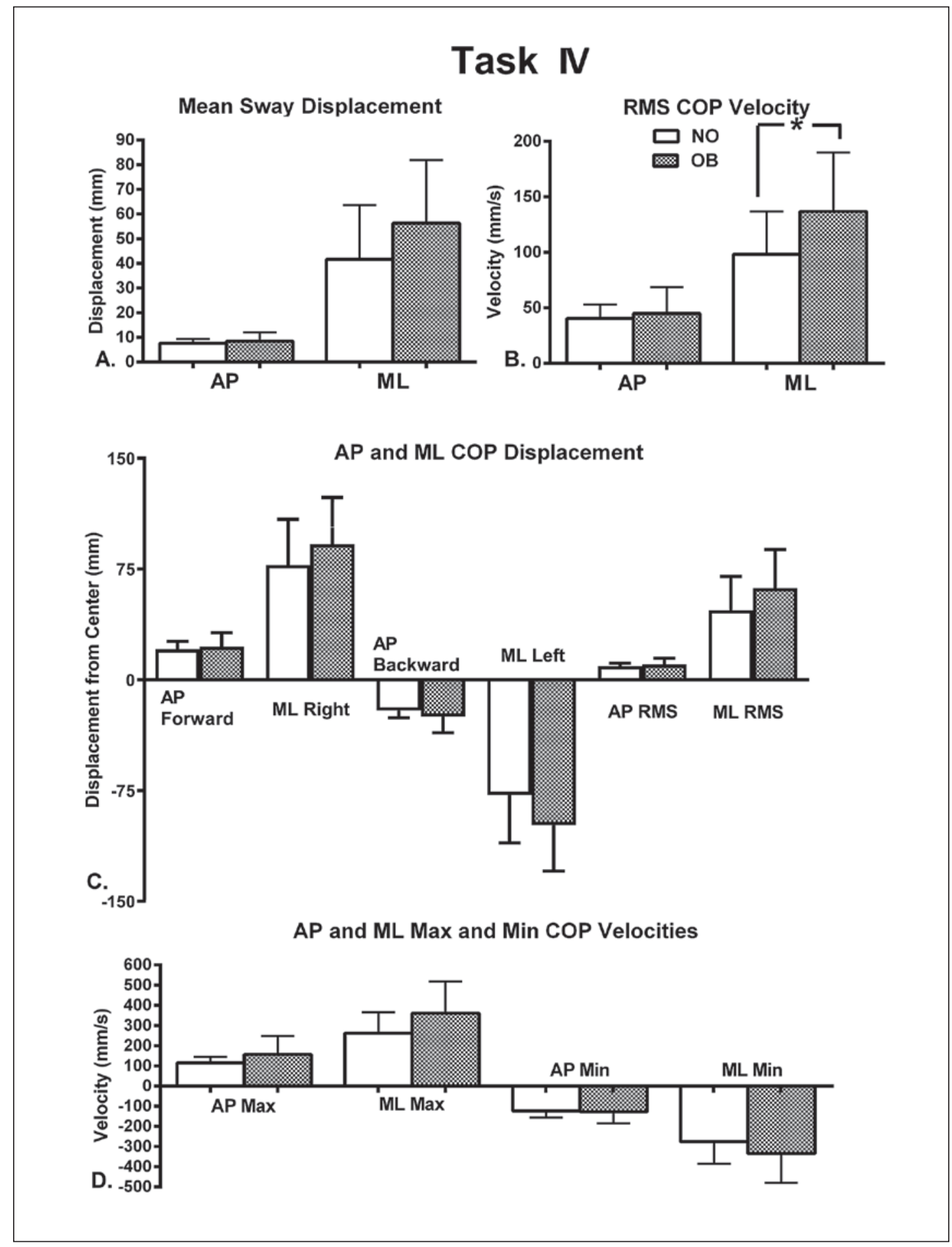

Fig. 5. Task IV force plate measurements. Subjects were instructed to repeatedly sway from left to right along the ML axis of the force plate while keeping eyes open. While AP displacements (A, C) and velocities (B, D) were not different between groups, paralleling the outcomes observed in task III, obese (OB) subjects appeared susceptible to deviations in the ML direction, despite the direction or degree of torso motion. Data are presented as mean \pm standard deviation (nonobese [NO]: $n=13$; OB: $n=17$ ). ${ }^{*} p<0.05$ (two-tailed Student $t$-test). AP, anteroposterior; COP, center of pressure; ML, mediolateral; RMS, root mean square. 
Pagnotti et al.: Postural Instability in Bariatric Patients

$(p<0.02)$ lower in magnitude for obese subjects than for nonobese ones, which translated to decreases of $30 \%(p<0.05)$ and $32 \%(p<0.03)$, respectively, in the maximum and minimum ML COP velocity of obese subjects. No differences in maximum or minimum AP COP velocities were observed.

Task IV (Dynamic Stance ML Sway: Eyes Open) (Fig. 5). Obese subjects were able to sway in the ML direction to the same extent as the nonobese ones when instructed. No differences in mean sway displacement or COP displacement along either axis was observed. However, $\mathrm{V}_{\mathrm{RMS}}$ measured along the ML direction was $40 \%(p<0.04)$ greater for obese subjects than for nonobese ones. No significant differences in maximum or minimum $V_{\mathrm{RMS}}$ were observed in either direction between groups.

\section{Discussion}

Increased fall and fracture risk in obese individuals can be attributed to greater instability caused by a greater moment of inertia about the torso complemented by waning muscle mass [46-49] and changes in posture and gait [50]. Further, limited mobility in obese individuals accelerates physiological repercussions through increased adipose burden and intramuscular fat, reducing muscle quality and tone. Indeed, increased sway length and sway velocity about a COP are presumed identifiers of at-risk populations [37]. Quantifying fall risk in obese patients is important, but most studies have focused on obese patients with a broad BMI range (BMI $>30 \mathrm{~kg} / \mathrm{m}^{2}$ ). Hence, our study addressed morbidly obese (BMI $\geq 40 \mathrm{~kg} / \mathrm{m}^{2}$ ) subjects, who were candidates elected for various bariatric procedures, and examined for differences in postural stability about their COP during a series of static (both with and without visual stimuli) and dynamic mobility tasks, with the latter employing upper torso movement with the lower extremities engaged for stability.

Since visual stimuli influence balance [51,52], COP stability was assessed with subjects' eyes either open or closed while sustaining a static footing position. The purpose was to elucidate at what speed and magnitude obese subjects deviate from a normalized COP then at what rate they return to that position while maintaining a fixed stance, and to determine if their stability is disabled when their eyes are closed. Our data show that obese subjects exhibit greater COP displacement about zero $(x=0, y=0)$ on the force plate, meaning that the circumferential range around the COP is greater than in nonobese subjects. Further, when subjects are instructed to remain static, this effect occurs significantly more in both the AP and ML directions. The velocity at which obese subjects reach this displacement is greater along the AP axis. Together, these data suggest that, dictated by these conditions, obese individuals risk greater instability, possibly attributable to inadequate stabilization musculature around their COP. These data align with findings from others' studies in the field suggesting instability in obese patients due to greater sway and velocity $[41,42]$. However, some contention exists in the interpretation of sway data in the sense that reduced sway and decreased velocity equate to great fall risk [13]. Importantly, task I did not incorporate mobility, hence voluntary motion was not necessary for instability to manifest.

In contrast to the previous task, task II stipulated that subjects keep their eyes closed. Results from task II indicated that the degree of sway displacement for obese subjects had twice the displacement along the AP axis than that observed in task I. In fact, significantly greater sway along the AP axis was observed both fore and aft of the neutral COP, implying that instability arose irrespective of visual cues. Sway displacement along the ML direction was unaffected. The degree to which sway deviated from neutral $(x=0, y=0)$ can be attributed to the greater $V_{\text {RMS }}$ (along the AP axis) observed in obese subjects in contrast to nonobese ones. Therefore, subjects afflicted by obesity, and others whose obesity is compounded by

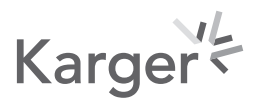


Pagnotti et al.: Postural Instability in Bariatric Patients

impaired visual or vestibular systems, could risk perpetuating injury from unrecoverable motion dynamics.

Tasks III and IV involved voluntary, dynamic motions to assess how subjects remained balanced while moving within their perceived zone of comfort. Other published work has employed dynamic tests to reveal measures of instability in obese populations [34]. Dynamic motion in task III did not yield differences in the displacement in the ML direction, most likely due to the dedicated fore and aft motions along the AP axis; however, it is interesting that $\mathrm{V}_{\mathrm{RMS}}$ in the ML direction was significantly lower in the obese subjects even though they were moving in the AP direction, perhaps a compensatory technique to maintain stability. This outcome further stresses the impaired mobility of obese subjects when challenged to use their stabilization muscles, and by slowing down. Different outcomes were observed in task $I V$, when subjects were asked to actively sway laterally, in that $V_{\text {RMS }}$ for obese subjects was significantly greater along the ML axis than for nonobese ones, even though both groups achieved the same sway displacement.

The findings we observed may be attributed to multiple factors, such as body composition or pharmaceutical influence. Type II fast-twitch fibers, responsible for stabilizing posture and coordinating shifts in balance, are compromised following high-fat diets and decreased physical exertion $[53,54]$. Contrasting individuals with similar BMI could delineate the impact of COP movements and stability due to body mass versus muscle and fat distribution. Limb girth and abdominal fat could independently drive specific variations in stability. Quantifying muscular strength, muscle fiber integrity, and the effect of visual stimuli on balance and movements about the COP would strengthen our conclusions and help determine to what magnitude stabilizing muscles are impaired in the obese phenotype. Including physical activity as a nonpharmacological modality to engage and maintain musculoskeletal strength while being equally responsible for suppressing visceral adiposity could improve stability in patients with obesity $[23,55]$. Nevertheless, incorporating elements of physical activity could drive improvements in mobility, stability (via balance), and proprioception by maintaining muscle mass while decreasing systemic fat. However, it is important to consider the means and degrees by which individual weight loss is achieved (certain bariatric interventions or pathological conditions). A subsequent reduction in muscle mass could also compromise stability due to functional and physiological alterations in musculoskeletal composition, center of gravity, and coordination [56].

To compound the physiological impact of obesity as contributory to falls, pharmacological interventions commonly prescribed to bariatric patients, such as tricyclic antidepressants (i.e., doxepin) [57] and antihypertensives (i.e., perindopril) [58], exhibit side effects that induce postural instability. The former acts on the central nervous system to inhibit neurotransmitter activity and signaling activity between the brain stem and spinal cord, while the latter slows hemodynamic responses; thus, both classes of drugs can exacerbate fall risk through impaired nervous feedback mechanisms or inducing postural hypotension. Contrasting evidence has shown that discrepancies in risk for injury exist across the different classes of obesity, with morbidly obese patients being at a potentially lower risk than their less obese (class I and II) counterparts [5]. Despite these findings, recent studies have refuted the overall protective nature of excess adiposity against injury [59].

One of the limitations of this study could be due to the variation in subject-perceived degree of comfort reported during tasks III and IV, causing the obese subjects to perform differently than if given a fixed distance. Despite this limitation, this provided insight into how obese patients would alter the velocity of their balancing motions to achieve the same extent of movement as the nonobese cohort. Additionally, even though the study excluded subjects who required the aid of a prosthetic, wheel chair, walker, or cane, we were not provided an exhaustive subject medical history; therefore, any prior physical disabilities (i.e., knee joint

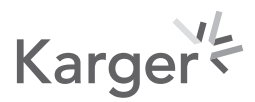




\section{Obesity Facts}

Pagnotti et al.: Postural Instability in Bariatric Patients

pain or muscle injuries) were unknown to those performing the evaluation except for subjectvolunteered information. Identifying key predictors of fall risk susceptibility could support the additional presurgical patient assessment in the obese population.

\section{Conclusion}

Stability measured via static and dynamic tasks demonstrated that preoperative obese bariatric patients who are morbidly obese exhibit reduced postural stability compared to nonobese individuals. Furthermore, these data highlight the negative impact of obesity on balance and underscore the importance of directing clinical attention towards improving stability in obese patients, specifically those elected for bariatric procedures. By uncovering the physical attributes that govern obesity-related instability, treatment modalities and physical therapies could be developed to minimize instability and mitigate injury risk during daily locomotion.

\section{Acknowledgments}

The authors would like to extend their appreciation to the staff at the Stony Brook Medicine's Division of Bariatric, Foregut and Advanced Gastrointestinal Surgery and the Stony Brook University Department of Biomedical Engineering for subject recruitment, facilitating data acquisition, and for the resources necessary to complete these assessments.

\section{Statement of Ethics}

This research followed the guidelines for human studies and includes evidence that the research was conducted ethically in accordance with the World Medical Association Declaration of Helsinki. Subject participation and evaluation protocols were approved by the Institutional Review Board Human Subjects committee at Stony Brook University. Subjects who volunteered were provided documentation outlining the study objectives, the tasks to be performed, and the option to opt out at any point during the study. Procedures as well as risks and benefits were explained to each consenting subject. Subjects who agreed to participate were given a copy of their signed consent form. No compensation was provided for study participation.

\section{Conflict of Interest Statement}

C.T. Rubin is a cofounder of Marodyne Medical, LLC and has several patents related to the ability of mechanical signals to control metabolic disorders. A.D. Pryor is a speaker for Ethicon Endo-Surgery Inc., Gore Industries, and Medtronic, and is a consultant for Apollo Endosurgery and Intuitive Surgical Inc. The other authors have no competing interests to declare. 
Pagnotti et al.: Postural Instability in Bariatric Patients

\section{Funding Sources}

This research did not receive any specific grant from funding agencies in the public, commercial, or not-for-profit sectors. Materials, resources, and facilities for this work were provided by the Stony Brook University Department of Biomedical Engineering and the Bariatric and Metabolic Weight Loss Center at Stony Brook Medicine.

\section{Author Contributions}

All authors provided substantial contribution to the design and implementation of this study and to the generation of the manuscript. The contributions of each author are as follows: G.M. Pagnotti, A.D. Pryor, K.E. Cottell, and M.E. Chan conceived the study design. G.M. Pagnotti, A. Yang, M.E. Chan, and K.E. Cottell acquired the data. G.M. Pagnotti, A. Haider, A. Yang, C.T. Rubin, and M.E. Chan analyzed the data and interpreted the findings. G.M. Pagnotti, A. Haider, K.E. Cottell, C.M. Tuppo, A.D. Pryor, C.T. Rubin, and M.E. Chan drafted the manuscript. G.M. Pagnotti, A. Haider, A. Yang, K.E. Cottell, C.M. Tuppo, K.-Y. Tong, A.D. Pryor, C.T. Rubin, and M.E. Chan provided critical revisions and final approval of the version submitted.

\section{References}

1 Thibaud M, Bloch F, Tournoux-Facon $\mathrm{C}$, Brèque $\mathrm{C}$, Rigaud AS, Dugué B, et al. Impact of physical activity and sedentary behaviour on fall risks in older people: a systematic review and meta-analysis of observational studies. Eur Rev Aging Phys Act. 2012 Apr;9(1):5-15.

2 Centers for Disease Control and Prevention. Falls are leading cause of injury and death in older Americans. 2016. Accessible online at: www.cdc.gov/media/releases/2016/p0922-older-adult-falls.html.

3 World Health Organization. Falls. 2017. Accessible online at: www.who.int/mediacentre/factsheets/fs344/ en/.

4 Fjeldstad C, Fjeldstad AS, Acree LS, Nickel KJ, Gardner AW. The influence of obesity on falls and quality of life. Dyn Med. 2008 Feb;7(1):4.

5 Himes CL, Reynolds SL. Effect of obesity on falls, injury, and disability. J Am Geriatr Soc. 2012 Jan;60(1):124-9.

6 Mitchell RJ, Lord SR, Harvey LA, Close JC. Associations between obesity and overweight and fall risk, health status and quality of life in older people. Aust N Z J Public Health. 2014 Feb;38(1):13-8.

7 Slemenda C, Heilman DK, Brandt KD, Katz BP, Mazzuca SA, Braunstein EM, et al. Reduced quadriceps strength relative to body weight: a risk factor for knee osteoarthritis in women? Arthritis Rheum. 1998 Nov;41(11): 1951-9.

8 Newcomer BR, Larson-Meyer DE, Hunter GR, Weinsier RL. Skeletal muscle metabolism in overweight and post-overweight women: an isometric exercise study using (31)P magnetic resonance spectroscopy. Int J Obes Relat Metab Disord. 2001 Sep;25(9):1309-15.

9 Sartorio A, Lafortuna CL, Conte G, Faglia G, Narici MV. Changes in motor control and muscle performance after a short-term body mass reduction program in obese subjects. J Endocrinol Invest. 2001 Jun;24(6):393-8.

10 Ochi M, Tabara Y, Kido T, Uetani E, Ochi N, Igase M, et al. Quadriceps sarcopenia and visceral obesity are risk factors for postural instability in the middle-aged to elderly population. Geriatr Gerontol Int. 2010 Jul;10(3): 233-43.

11 Igase M, Tabara Y, Ochi M, Kohara K, Miki T. Sarcopenia and visceral obesity: implications for postural instability in the elderly population. Nihon Ronen Igakkai Zasshi. 2011;48(1):47-50. Japanese.

12 Simoneau M, Teasdale N. Balance control impairment in obese individuals is caused by larger balance motor commands variability. Gait Posture. 2015 Jan;41(1):203-8.

13 Carneiro JA, Santos-Pontelli TE, Vilaça KH, Pfrimer K, Colafêmina JF, Carneiro AA, et al. Obese elderly women exhibit low postural stability: a novel three-dimensional evaluation system. Clinics (São Paulo). 2012;67(5): 475-81.

14 Corbeil P, Simoneau M, Rancourt D, Tremblay A, Teasdale N. Increased risk for falling associated with obesity: mathematical modeling of postural control. IEEE Trans Neural Syst Rehabil Eng. 2001 Jun;9(2):126-36.

15 Sun F, Wang LJ, Wang L. Effects of weight management program on postural stability and neuromuscular function among obese children: study protocol for a randomized controlled trial. Trials. 2015 Apr;16(1):143.

16 World Health Organization. Obesity and overweight. 2016. Accessible online at: www.who.int/dietphysicalactivity/media/en/gsfs_obesity.pdf. 
Pagnotti et al.: Postural Instability in Bariatric Patients 2011-2014. NCHS Data Brief. 2015 Nov;219:1-8.

18 Centers for Disease Control and Prevention. Defining adult overweight and obesity. 2020. Accessible online at: www.cdc.gov/obesity/adult/defining.html.

19 Zhu C, Pryor AD. Innovations in Bariatric Surgery. Surg Technol Int. 2015 Nov;27:129-35.

20 Forhan M, Gill SV. Obesity, functional mobility and quality of life. Best Pract Res Clin Endocrinol Metab. 2013 Apr;27(2):129-37.

21 Grotle M, Hagen KB, Natvig B, Dahl FA, Kvien TK. Obesity and osteoarthritis in knee, hip and/or hand: an epidemiological study in the general population with 10 years follow-up. BMC Musculoskelet Disord. 2008 0ct; $9(1)$ : 132.

22 Hertel J. Functional Anatomy, Pathomechanics, and Pathophysiology of Lateral Ankle Instability. J Athl Train. 2002 Dec;37(4):364-75.

23 Maffiuletti NA, Agosti F, Proietti M, Riva D, Resnik M, Lafortuna CL, et al. Postural instability of extremely obese individuals improves after a body weight reduction program entailing specific balance training. J Endocrinol Invest. 2005 Jan;28(1):2-7.

24 Livhits M, Mercado C, Yermilov I, Parikh JA, Dutson E, Mehran A, et al. Exercise following bariatric surgery: systematic review. Obes Surg. 2010 May;20(5):657-65.

25 Kim D, Gill SV. Changes in center of pressure velocities during obstacle crossing one year after bariatric surgery. Gait Posture. 2020 Feb;76:377-81.

26 Jacobi D, Ciangura C, Couet C, Oppert JM. Physical activity and weight loss following bariatric surgery. Obes Rev. 2011 May;12(5):366-77.

27 Visser JE, Carpenter MG, van der Kooij H, Bloem BR. The clinical utility of posturography. Clin Neurophysiol. 2008 Nov; 119(11):2424-36.

28 Colledge NR, Cantley P, Peaston I, Brash H, Lewis S, Wilson JA. Ageing and balance: the measurement of spontaneous sway by posturography. Gerontology. 1994;40(5):273-8.

29 Soto-Varela A, Faraldo-García A, Rossi-Izquierdo M, Lirola-Delgado A, Vaamonde-Sánchez-Andrade I, del-RíoValeiras M, et al. Can we predict the risk of falls in elderly patients with instability? Auris Nasus Larynx. 2015 Feb;42(1):8-14.

30 Howcroft J, Lemaire ED, Kofman J, McIlroy WE. Elderly fall risk prediction using static posturography. PLoS One. 2017 Feb;12(2):e0172398.

31 Rossi-Izquierdo M, Basta D, Rubio-Rodríguez JP, Santos-Pérez S, Ernst A, Sesar-Ignacio Á, et al. Is posturography able to identify fallers in patients with Parkinson's disease? Gait Posture. 2014;40(1):53-7.

32 Rogers JH. Romberg and his test. J Laryngol Otol. 1980 Dec;94(12):1401-4.

33 Handrigan G, Hue O, Simoneau M, Corbeil P, Marceau P, Marceau S, et al. Weight loss and muscular strength affect static balance control. Int J Obes. 2010 May;34(5):936-42.

34 Bataweel EA, Ibrahim AI. Balance and musculoskeletal flexibility in children with obesity: a cross-sectional study. Ann Saudi Med. 2020 Mar-Apr;40(2):120-5.

35 Shultz SP, Sitler MR, Tierney RT, Hillstrom HJ, Song J. Consequences of pediatric obesity on the foot and ankle complex. J Am Podiatr Med Assoc. 2012 Jan-Feb;102(1):5-12.

36 Grella R, Del Torto G, Nicoletti GF, D’Andrea F. Postural Changes After Abdominoplasty in Morbid Obese Patients. Ann Plast Surg. 2019 Jul;83(1):89-93.

37 Wearing SC, Hennig EM, Byrne NM, Steele JR, Hills AP. The biomechanics of restricted movement in adult obesity. Obes Rev. 2006 Feb;7(1):13-24.

38 Boucher F, Handrigan GA, Mackrous I, Hue O. Childhood obesity affects postural control and aiming performance during an upper limb movement. Gait Posture. 2015 Jul;42(2):116-21.

39 Berg WP, Alessio HM, Mills EM, Tong C. Circumstances and consequences of falls in independent communitydwelling older adults. Age Ageing. 1997 Jul;26(4):261-8.

40 Piirtola M, Era P. Force platform measurements as predictors of falls among older people - a review. Gerontology. 2006;52(1):1-16.

41 Rezaeipour M. Evaluation of postural stability in overweight and obese middle-aged men. Turk J Med Sci. 2018 Oct;48(5):1053-7.

42 Lockhart TE, Frames CW, Soangra R, Lieberman A. Effects of Obesity and Fall Risk on Gait and Posture of Community-Dwelling Older Adults. Int J Progn Health Manag. 2019;10(1):019.

43 Gurfinkel EV. Physical foundations of stabilography. Agressologie. 1973 Sep;14(Spec No C):9-13.

44 Maurer C, Peterka RJ. A new interpretation of spontaneous sway measures based on a simple model of human postural control. J Neurophysiol. 2005 Jan;93(1):189-200.

45 Muir J, Judex S, Qin YX, Rubin C. Postural instability caused by extended bed rest is alleviated by brief daily exposure to low magnitude mechanical signals. Gait Posture. 2011 Mar;33(3):429-35.

46 Huang RP, Rubin CT, McLeod KJ. Changes in postural muscle dynamics as a function of age. J Gerontol A Biol Sci Med Sci. 1999 Aug;54(8):B352-7.

47 Laughton CA, Slavin M, Katdare K, Nolan L, Bean JF, Kerrigan DC, et al. Aging, muscle activity, and balance control: physiologic changes associated with balance impairment. Gait Posture. 2003 0ct;18(2):101-8.

48 Berry SD, Miller RR. Falls: epidemiology, pathophysiology, and relationship to fracture. Curr Osteoporos Rep. 2008 Dec;6(4):149-54. 
Pagnotti et al.: Postural Instability in Bariatric Patients

49 Rossi-Izquierdo M, Santos-Pérez S, Faraldo-García A, Vaamonde-Sánchez-Andrade I, Gayoso-Diz P, Del-RíoValeiras M, et al. Impact of obesity in elderly patients with postural instability. Aging Clin Exp Res. 2016 Jun; 28(3):423-8.

50 Gonzalez M, Gates DH, Rosenblatt NJ. The impact of obesity on gait stability in older adults. J Biomech. 2020 Feb;100:109585.

51 Reason J, Wagner H, Dewhurst D. A visually-driven postural after-effect. Acta Psychol (Amst). 1981 Aug;48(13):241-51.

52 Collins JJ, De Luca CJ. The effects of visual input on open-loop and closed-loop postural control mechanisms. Exp Brain Res. 1995;103(1):151-63.

53 Ciapaite J, van den Berg SA, Houten SM, Nicolay K, van Dijk KW, Jeneson JA. Fiber-type-specific sensitivities and phenotypic adaptations to dietary fat overload differentially impact fast- versus slow-twitch muscle contractile function in C57BL/6J mice. J Nutr Biochem. 2015 Feb;26(2):155-64.

54 Eshima H, Tamura Y, Kakehi S, Kurebayashi N, Murayama T, Nakamura K, et al. Long-term, but not short-term high-fat diet induces fiber composition changes and impaired contractile force in mouse fast-twitch skeletal muscle. Physiol Rep. 2017 Apr;5(7):e13250.

55 Teasdale N, Hue O, Marcotte J, Berrigan F, Simoneau M, Doré J, et al. Reducing weight increases postural stability in obese and morbid obese men. Int J Obes. 2007 Jan;31(1):153-60.

56 Hue O, Berrigan F, Simoneau M, Marcotte J, Marceau P, Marceau S, et al. Muscle force and force control after weight loss in obese and morbidly obese men. Obes Surg. 2008 Sep;18(9):1112-8.

57 Li X, Hamdy R, Sandborn W, Chi D, Dyer A. Long-term effects of antidepressants on balance, equilibrium, and postural reflexes. Psychiatry Res. 1996 Jul;63(2-3):191-6.

58 Sumukadas D, Price R, McMurdo ME, Rauchhaus P, Struthers A, McSwiggan S, et al. The effect of perindopril on postural instability in older people with a history of falls - a randomised controlled trial. Age Ageing. 2018 Jan;47(1):75-81.

59 Compston JE, Watts NB, Chapurlat R, Cooper C, Boonen S, Greenspan S, et al.; Glow Investigators. Obesity is not protective against fracture in postmenopausal women: GLOW. Am J Med. 2011 Nov;124(11):1043-50. 